\title{
Duration of wrinkle correction following repeat treatment with Juvéderm hyaluronic acid fillers
}

\author{
Stacy R. Smith $\cdot$ Derek Jones $\cdot$ Jane A. Thomas $\cdot$ \\ Diane K. Murphy $\cdot$ Frederick C. Beddingfield III
}

Received: 6 May 2010/Revised: 23 September 2010/Accepted: 24 September 2010/Published online: 9 October 2010

(C) The Author(s) 2010. This article is published with open access at Springerlink.com

\begin{abstract}
Many patients elect to have repeat treatments with hyaluronic acid dermal fillers to maintain wrinkle correction, but the clinical performance of these products after repeat treatments has not been formally assessed. The primary objective of this study was to evaluate the effectiveness of Juvéderm injectable gel (Juvéderm Ultra, Juvéderm Ultra Plus, and Juvéderm 30) through 1 year after repeat treatment of nasolabial folds (NLFs) that were previously treated with Juvéderm or Zyplast 6-9 months prior to the repeat treatment. Upon completion of the pivotal IDE clinical trial for Juvéderm, five of the original 11 study sites were selected to participate in an extended follow-up evaluation, and a total of 80 subjects were enrolled. For the Juvéderm-treated NLFs in each treatment group, the median injection volume was $1.5-1.6 \mathrm{~mL}$ for initial
\end{abstract}

Originally presented as a poster at the Academy 2007 meeting of the American Academy of Dermatology, August 1-5, 2007.

\section{S. R. Smith $(\bowtie)$}

Therapeutics Clinical Research, Inc., 9025 Balboa Avenue \#105, San Diego, CA 92123, USA

e-mail: ssmith@therapeuticsresearch.com

\section{S. R. Smith}

Division of Dermatology, University of California,

San Diego, San Diego, USA

D. Jones

Skin Care and Laser Physicians of Beverly Hills,

9201 W. Sunset Blvd., Suite 602, Los Angeles,

CA 90069, USA

D. Jones · F. C. Beddingfield III

Division of Dermatology, David Geffen School of Medicine,

University of California, Los Angeles, Los Angeles, USA

J. A. Thomas · D. K. Murphy · F. C. Beddingfield III

Allergan, Santa Barbara, CA, USA treatment but only $0.5-0.6 \mathrm{~mL}$ for the repeat treatment $(p<0.0001)$. Mean Investigator-assigned NLF severity scores on a scale of $0-4$ for the Juvéderm-treated NLFs improved from 2.5-2.7 (moderate to severe) at baseline to $1.2-1.5$ (mild) just prior to repeat treatment ( $>24$ weeks) and $0.7-0.9$ (mild) at 4 weeks after repeat treatment. At 48 weeks post-repeat treatment, the mean NLF scores were 1.1-1.3 (mild), and $78-90 \%$ of subjects were considered responders ( $\geq 1$ point improvement). Thus, subjects sustained a total of 18-21 months of wrinkle correction with a repeat treatment at 6-9 months and needed substantially less filler $(60 \%$ less $)$ for repeat treatment than for initial treatment, indicating that retreatment at this timepoint may be beneficial to patients.

Keywords Dermal filler - Wrinkles - Hyaluronic acid · Cosmetic techniques $\cdot$ Follow-up studies

\section{Introduction}

Dermal fillers serve as one of the most common and useful treatments for wrinkles and folds. Hyaluronic acid products are the most widely used in the U.S. and enjoy a favorable risk/benefit profile. These products provide temporary correction of the treated area with eventual resorption of the material and a presumed return to the patient's pretreatment state. Many patients elect to have repeat treatments to maintain the correction; however, the clinical performance after repeat treatments has not been formally assessed for most filler products.

The primary objective of this follow-up study was to evaluate the effectiveness of Juvéderm injectable gel (Juvéderm Ultra, Juvéderm Ultra Plus, and Juvéderm 30) through 1 year after repeat treatment of nasolabial folds 
(NLFs) that were previously treated with Juvéderm or Zyplast 6-9 months prior to the repeat treatment. An additional effectiveness objective was to determine if the volume of Juvéderm injected at repeat treatment was different from the volume injected during the initial treatment period and to determine the duration of improvement in NLF severity after repeat treatment.

\section{Methods}

\section{Study design}

Upon completion of the pivotal IDE clinical trial for Juvéderm 30, Ultra, and Ultra Plus, five of the original 11 study sites were selected to participate in an extended follow-up evaluation. Sites were selected based on their continued abilities to participate in the follow-up protocol, their track record of visit schedule compliance, and the planned sample size of 150 subjects. No consideration was given to duration of filler correction in the selection of sites. Subjects who were eligible and agreed to participate in the follow-up study signed an informed consent and were followed from 4 through 48 weeks after their repeat treatments. Routine follow-up visits for effectiveness occurred at 4, 12, and 24 weeks, and an amendment to the protocol added visits at 36 and 48 weeks after repeat treatment. Safety and effectiveness were evaluated at each office visit.

Subjects were enrolled in this follow-up study if they had completed the pivotal study, preferred the Juvéderm-treated side (versus the Zyplast-treated side) upon study exit and had undergone their optional end-of-study (repeat) treatment to both NLFs on the same day and with the same Juvéderm formulation as was administered during the pivotal trial. The repeat treatment was administered between 24 and 36 weeks ( \pm 14 days) after the last treatment in the pivotal study.

Subjects were excluded from the study if they had facial hair that would interfere with the visual assessments of NLF severity; had undergone or had plans to undergo any confounding esthetic procedure such as botulinum toxin injection, laser resurfacing, etc., in the lower two-thirds of the face less than 30 days prior to the repeat treatment or at any time thereafter through the end of the study; had a clinically significant organic disease, condition, illness, or circumstance that would compromise participation in the trial; or had received any other investigational treatment within 30 days prior to study enrollment.

\section{Treatment}

During the pivotal trial, subjects were randomized to receive a single formulation of Juvéderm in one NLF and Zyplast collagen in the opposite NLF. The three Juvéderm formulations all have a hyaluronic acid concentration of $24 \mathrm{mg} / \mathrm{mL}$ and are manufactured using the same technique (Hylacross technology). Juvéderm Ultra can be injected through a $30 \mathrm{G}$ needle to allow for more versatility in treating wrinkles, whereas Juvéderm 30 is a thicker product that can be injected through a $27 \mathrm{G}$ needle for contouring deeper folds. Juvéderm Ultra Plus has the highest degree of crosslinking of the three fillers to allow extended correction of deeper folds. An initial treatment and up to two touch-up treatments at 2-week intervals after initial treatment were performed to achieve optimal correction in both NLFs and subjects were followed through 24 weeks after the last treatment. At the end of the pivotal study, subjects were asked which treatment they preferred and were subsequently unblinded. Subjects were offered repeat treatment of both NLFs with the original Juvéderm formulation after the 24-week visit. For this follow-up study, subjects remained non-randomized and unblinded.

Investigators determined the appropriate volume of Juvéderm needed to obtain optimal correction at the initial, touch-up, and repeat treatments. The same formulation for Juvéderm (Ultra, Ultra Plus, or 30) was used for the initial and repeat treatments. Repeat treatment took place in a single session with no limit on injection volume, and no additional treatments were administered in the follow-up study.

\section{Outcome measures}

Effectiveness evaluations were based on NLF severity assessments and the volume $(\mathrm{mL})$ of filler injected. Injection volumes were compared for the initial and repeat treatments, and NLF severity was evaluated throughout the follow-up periods. The Investigator used the same validated 5-point photographic wrinkle assessment scale (WAS, Table 1) as was used in the pivotal trial to make live assessments of the severity of the subject's NLFs. The scale represents the spectrum of NLF severity from least to most severe (range 0-4) and has reference photographs for each severity grade. The subjects used the same 5-point scale as the Investigator, except that the subjects made their self assessments by using the written and numerical descriptions for reference while examining their NLFs in a mirror.

Table 1 5-Point Wrinkle Assessment Scale for NLF severity

\begin{tabular}{lll}
\hline Score & Severity descriptions \\
\hline 4 & Extreme & $\begin{array}{c}\text { Very deep wrinkle, redundant fold } \\
\text { (overlapping skin) } \\
\text { Deep wrinkle, well-defined edges } \\
\text { (but not overlapping) }\end{array}$ \\
2 & Severe & Moderately deep wrinkle \\
1 & Moderate & Shallow, just perceptible wrinkle \\
0 & Mild & No wrinkle \\
\hline
\end{tabular}


The Investigator also evaluated subjects for signs and symptoms of serious adverse events or unanticipated adverse events at each study visit. Serum samples were obtained from subjects at the 4-week post-repeat treatment visit for IgG antibody testing and comparison with baseline antibody titers.

\section{Statistical analyses}

Effectiveness analyses were performed on the intent-totreat population and safety analyses on the "as treated" population. A paired $t$ test was utilized to compare the volume at initial treatment with the volume at repeat treatment and the post-treatment NLF severity to baseline. Clinically significant improvement in NLF severity was defined a priori as $\geq 1$ point reduction in the WAS score. A $p$ value of $<0.05$ was considered statistically significant.

\section{Results}

Subjects

Of the planned 150 subjects at the five selected sites, 80 individuals signed consent forms and enrolled in the follow-up study (Fig. 1); 78 subjects completed 24 weeks of post-repeat treatment follow-up. The revised enrollment number from 150 to 80 subjects was due to a scarcity of subjects eligible for the follow-up study (i.e., subjects chose to delay repeat treatment beyond the 24-36-week treatment period window allowed in the study because additional correction was not yet needed). Following implementation of the protocol amendment adding followup visits at 36 and 48 weeks, 31 subjects who had not already passed those key timepoints consented to re-enroll, and $100 \%$ completed 48 weeks of post-repeat treatment follow-up. The primary reason for subjects not consenting to re-enroll for the extended follow-up was that they had already passed the 48-week timepoint when the protocol amendment was implemented.

A majority of the subjects in each cohort were Caucasian and female with a median age between 47 and 53 years (Table 2). More than one-third of subjects in each cohort had Fitzpatrick Skin Phototypes IV, V, or VI. The average interval from the last touch-up injection of the initial treatment until the repeat treatment injection was 201 days (range 149-275 days).

\section{Effectiveness}

The injection volume required to achieve optimal correction was significantly less $(p<0.0001)$ at repeat treatment than at initial treatment (Fig. 2). For the Juvéderm-treated

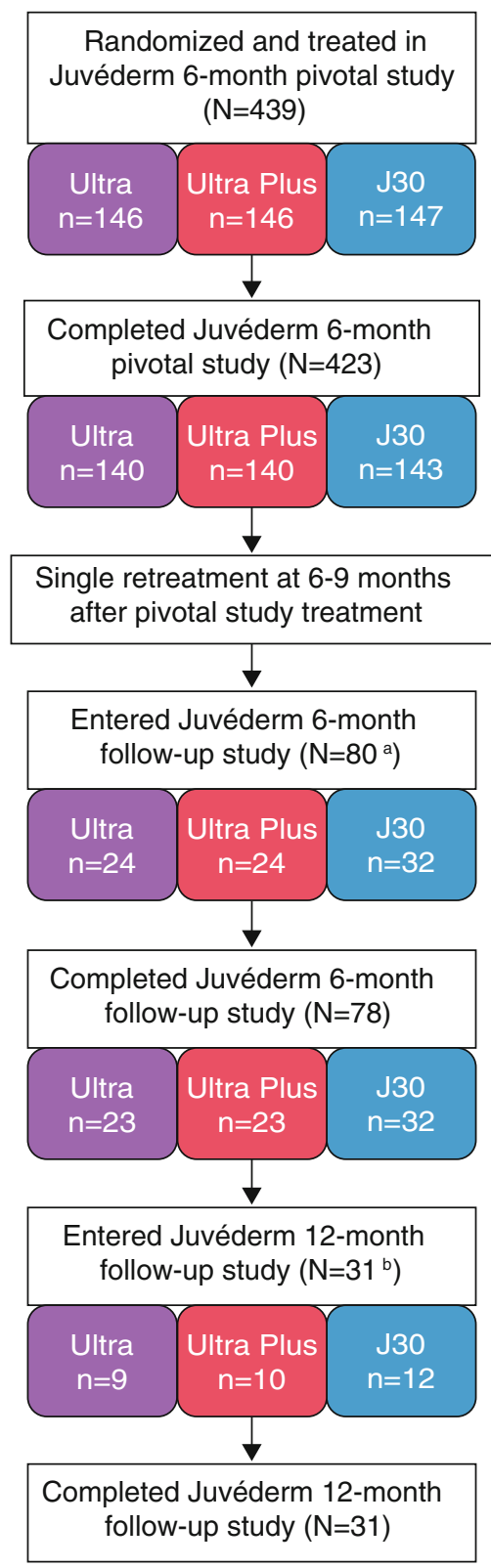

Fig. 1 Subject disposition flowchart. aplanned enrollment was 150 subjects. The primary reason that 150 was not achieved was that subjects were ineligible because they chose to delay repeat treatment beyond the window allowed in the Juvéderm follow-up study protocol. ${ }^{\mathrm{b}}$ The primary reason for subjects not re-enrolling in the extended portion of the Juvéderm follow-up study was that they had already passed the 6-month timepoint when the protocol amendment was implemented

NLFs in each treatment group, the median injection volume for initial treatment was $1.5-1.6 \mathrm{~mL}$, but only $0.5-0.6 \mathrm{~mL}$ was required for the repeat treatment depending upon which Juvéderm formulation was used. The median volumes of Zyplast at initial treatment were 2.3-2.8 $\mathrm{mL}$ (in each of the three Juvéderm formulation arms of the study); these previously Zyplast-treated folds 
Table 2 Subject demographics (ITT population)

\begin{tabular}{|c|c|c|c|c|c|c|}
\hline & \multicolumn{2}{|c|}{ Juvéderm Ultra $(N=24)$} & \multicolumn{2}{|c|}{ Juvéderm Ultra Plus $(N=24)$} & \multicolumn{2}{|c|}{ Juvéderm $30(N=32)$} \\
\hline & $N$ & Percentage & $N$ & Percentage & $N$ & Percentage \\
\hline \multicolumn{7}{|l|}{ Gender } \\
\hline Female & 21 & 88 & 22 & 92 & 28 & 88 \\
\hline Male & 3 & 13 & 2 & 8 & 4 & 13 \\
\hline \multicolumn{7}{|l|}{ Age (years) } \\
\hline Median & 48.5 & & 47.0 & & 52.5 & \\
\hline Range & $32-75$ & & $35-74$ & & $32-66$ & \\
\hline \multicolumn{7}{|l|}{ Ethnicity } \\
\hline Caucasian & 14 & 58 & 17 & 71 & 19 & 59 \\
\hline African American & 4 & 17 & 5 & 21 & 9 & 28 \\
\hline Hispanic & 4 & 17 & 2 & 8 & 3 & 9 \\
\hline Asian & 2 & 8 & 0 & 0 & 0 & 0 \\
\hline Other & 0 & 0 & 0 & 0 & 1 & 3 \\
\hline \multicolumn{7}{|c|}{ Fitzpatrick skin phototype } \\
\hline I & 0 & 0 & 3 & 13 & 0 & 0 \\
\hline II & 4 & 17 & 5 & 22 & 5 & 15 \\
\hline III & 10 & 42 & 6 & 26 & 8 & 24 \\
\hline IV & 5 & 21 & 5 & 22 & 10 & 30 \\
\hline $\mathrm{V}$ & 4 & 17 & 4 & 17 & 6 & 19 \\
\hline VI & 1 & 4 & 1 & 4 & 3 & 9 \\
\hline
\end{tabular}

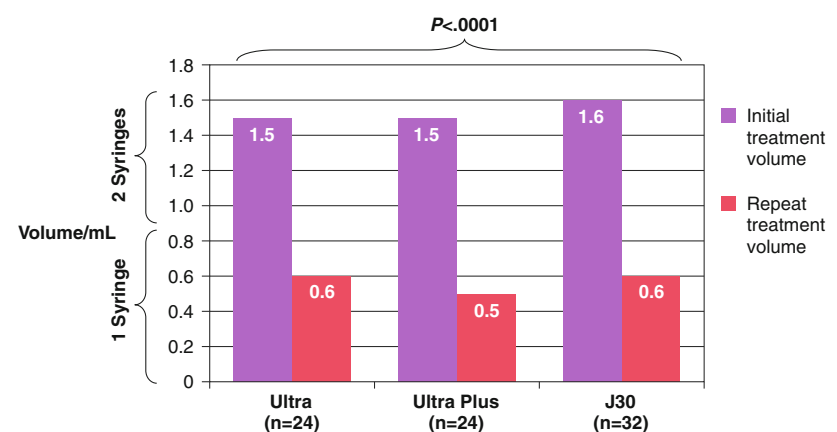

Fig. 2 Median injection volume at initial and repeat treatment

required a median amount of $1.0-1.2 \mathrm{~mL}$ of Juvéderm to achieve optimal correction at repeat treatment.

Mean Investigator-assigned NLF severity scores for the Juvéderm-treated NLFs remained improved from 2.5-2.7 (moderate to severe) at baseline to 1.2-1.5 (mild) just prior to repeat treatment (greater than 24 weeks) (Fig. 3). After repeat treatment, the mean NLF scores at 4 weeks were $0.7-0.9$ (mild), similar to the mean scores seen after completion of the initial treatment. At 48 weeks post-repeat treatment, the mean NLF WAS scores ranged from 1.1 to 1.3 (mild). Subject assessments paralleled those of Investigators, with improvement from mean scores of 2.3-2.5 at baseline to $0.8-0.9$ at 48 weeks post-repeat treatment.

The mean improvement in NLF severity remained clinically significant ( $\geq 1$ point) from 4 weeks after initial

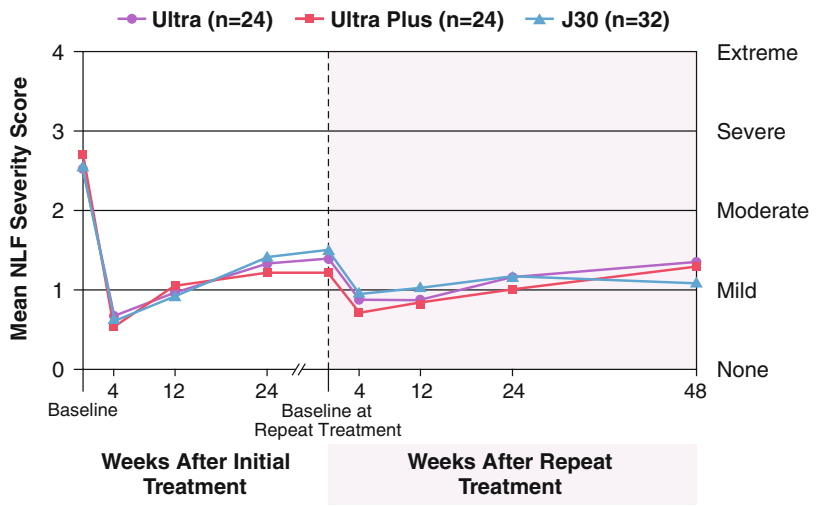

Fig. 3 Mean NLF severity score assessed by Investigators

treatment through 48 weeks after repeat treatment. Thus, subjects sustained a total of 18-21 months of wrinkle correction with a repeat treatment at 6-9 months (Fig. 4). Furthermore, a full $78-90 \%$ of subjects were responders ( $\geq 1$ point WAS improvement) at 48 weeks post-repeat treatment (Fig. 5), and the long-term results showed a smooth, natural looking wrinkle correction (Fig. 6).

Safety and tolerability

No serious or unanticipated adverse events were reported. One subject had positive serum IgG antibody titers at 


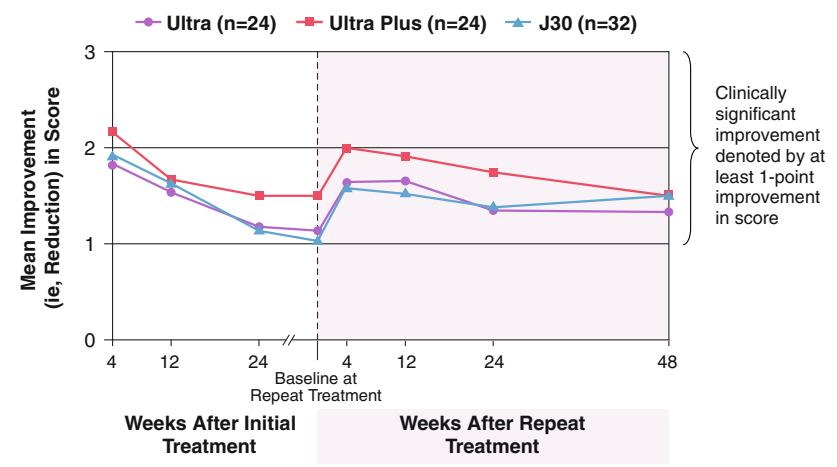

Fig. 4 Mean improvement from baseline in NLF severity score based on Investigator assessments

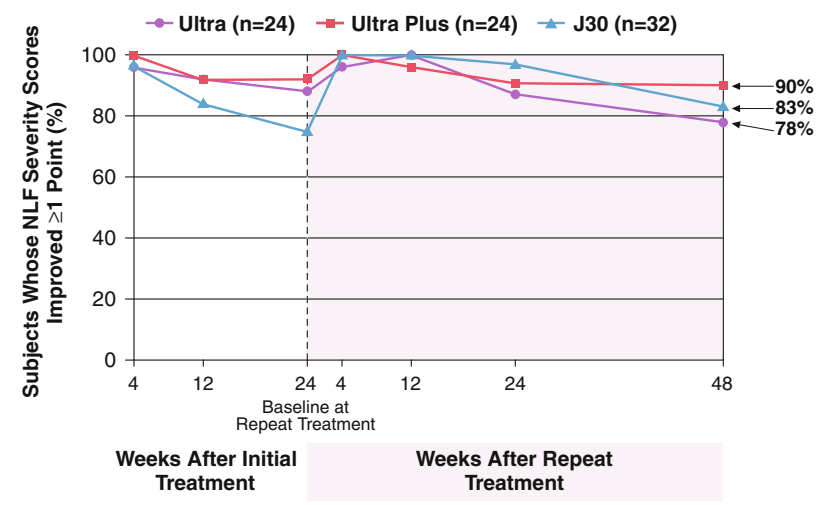

Fig. 5 Percent of subjects with improved NLF severity scores from initial baseline based on Investigator assessment
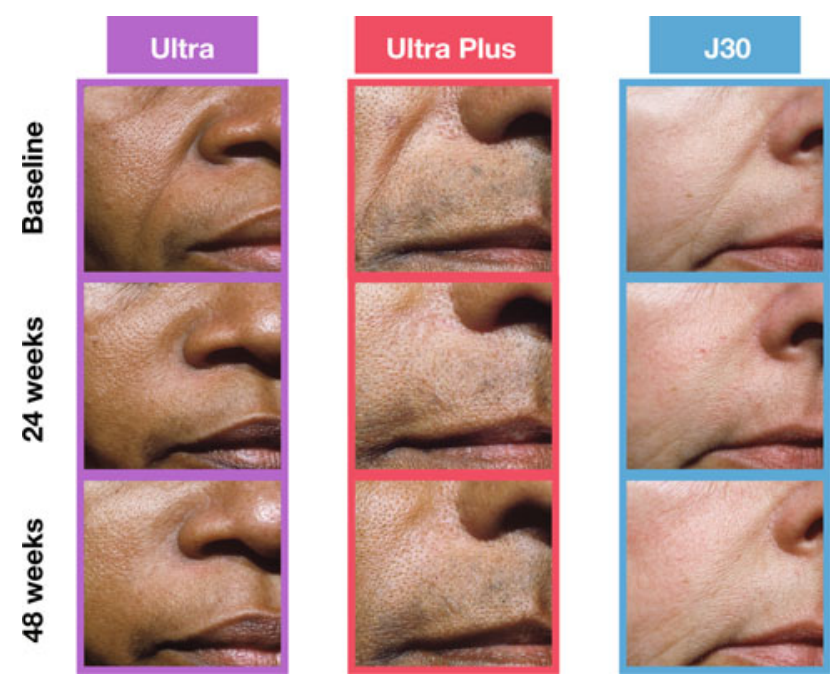

Fig. 6 Subjects demonstrating duration of effect with Ultra, Ultra Plus, and J30 dermal fillers at baseline and 24 and 48 weeks after repeat treatment
24 weeks after initial and 4 weeks after repeat treatment, but no clinical signs or symptoms of hypersensitivity.

\section{Discussion}

The impermanent nature of most dermal fillers and the progressive nature of skin aging require that the patient undergoes repeat treatments to maintain the desired correction. Typically, these are done when the patient notices that the prior correction has declined, additional correction is desired, and/or when finances allow. This study demonstrates that a repeat treatment performed at 6-9 months results in a prolonged benefit with a dramatic reduction in the volume of material required to achieve optimal correction and thus less impact on the patient's financial resources. Accordingly, it is likely in the patient's best interest for the physician to recommend re-injections at this timepoint even if significant correction is still seen from the initial injections. Re-injecting while some correction remains enables the patient to enjoy a very prolonged result which requires only a fraction of the amount of material required for the initial injections. Injecting a smaller volume while a significant correction remains also yields less dramatic changes in the appearance over time, leading to a natural appearance and making the fact that one has had treatments less noticeable to the public, which for many patients is a benefit. These effects for the 6-9-month reinjection appear to be specific to the Juvéderm products, however, as the subjects who were initially treated with Zyplast required substantially higher volumes of Juvéderm at their retreatment compared to subjects who had initially received hyaluronic acid.

Precisely, why such substantial differences are seen with re-injection is unknown. The elimination kinetics of crosslinked hyaluronic acid are not well characterized. Data from a single subject using high spatial resolution $T_{2}$ parametric magnetic resonance images showed that very small amounts of injected hyaluronic acid can be detected 4-9 months after injection into the forearm [2]. Wang et al. [5] demonstrated increased collagenesis in response to tissue expansion after dermal filler injection. This concept is further supported by a study of 63 subjects who received repeat treatment with Restylane in which Narins et al. [4] showed longevity of hyaluronic acid filler injection after retreatment and speculated that maintaining the tension from the tissue expansion stimulated collagen production and inhibited collagen breakdown.

It remains unclear whether the persistence of correction seen with longer follow-up in non-permanent filler materials is due to retained material or another phenomenon. While the actual mechanism of action for persistence is not clearly elucidated, the clinical endpoints seen in the 
grading scales used in studies of dermal fillers have been shown to be reproducible and reliable $[1,3]$. The results are real, regardless of how they occur.

Hyaluronic acid (as hyaluronan) is found in many tissues of the body and is generally considered innocuous when injected as a cross-linked polymer. Any time there are repeated exposures to foreign materials, the possibility of sensitization becomes a concern. In this study, only a single subject was noted to develop antibodies directed towards hyaluronic acid, and no clinical findings consistent with allergy or sensitization were noted in this subject.

This study was conducted as an addendum to the pivotal study for the approval of the Juvéderm family of products in the U.S. While the number of subjects participating in this longer-term study is smaller than in the original study, the sample size was large enough to obtain statistically significant results for the study endpoints. Although three formulations of Juvéderm (Juvéderm Ultra, Juvéderm Ultra Plus, and Juvéderm 30) were studied, only two of these formulations (Juvéderm Ultra and Ultra Plus) are commercially available in the U.S.

\section{Conclusions}

The Juvéderm family of fillers provides clinically significant improvement in the severity of nasolabial folds through 18-21 months in subjects who receive a repeat treatment at 6-9 months. Subjects need substantially less filler ( $60 \%$ less) for repeat treatment than for initial treatment, indicating that retreatment at this timepoint may be beneficial to the patient in terms of reduced price for smaller subsequent treatments and less dramatic swings in appearance. The Juvéderm fillers demonstrated effectiveness and safety across all skin types, as well as in both male and female subjects. Their extended persistence, resulting in less frequent treatments, combined with the natural look and feel of the skin post-treatment can be expected to produce a high level of patient satisfaction.

Acknowledgments The authors would like to thank Ramkumar Krish, MS, for biostatistical assistance and Allergan for financial support. Investigators participating in this study included Leslie Baumann, MD and Heather Woolery-Lloyd, MD (Miami, FL); Pearl Grimes, MD (Los Angeles, CA); Derek Jones, MD (Los Angeles, CA); Gary Monheit, MD (Birmingham, AL); and Stacy Smith, MD (San Diego, CA).

Conflict of interest Drs. Smith and Jones received research support for conducting this study. Ms. Thomas, Ms. Murphy, and Dr. Beddingfield are Allergan employees and stockholders.

Open Access This article is distributed under the terms of the Creative Commons Attribution Noncommercial License which permits any noncommercial use, distribution, and reproduction in any medium, provided the original author(s) and source are credited.

\section{References}

1. Day DJ, Littler CM, Swift RW, Gottlieb S (2004) The wrinkle severity rating scale: a validation study. Am J Clin Dermatol 5(1):49-52

2. Gensanne D, Josse G, Schmitt AM, Lagarde JM, Vincensini D (2007) In vivo visualization of hyaluronic acid injection by high spatial resolution T2 parametric magnetic resonance images. Skin Res Technol 13(4):385-389

3. Lemperle G, Holmes RE, Cohen SR, Lemperle SM (2001) A classification of facial wrinkles. Plast Reconstr Surg 108(6): 1735-1750 discussion 1751-1752

4. Narins RS, Dayan SH, Brandt FS, Baldwin EK (2008) Persistence and improvement of nasolabial fold correction with nonanimalstabilized hyaluronic acid 100,000 gel particles/mL filler on two retreatment schedules: results up to 18 months on two retreatment schedules. Dermatol Surg 34:S2-S8

5. Wang F, Garza LA, Kang S, Varani J, Orringer JS, Fisher GJ, Voorhees JJ (2007) In vivo stimulation of de novo collagen production caused by cross-linked hyaluronic acid dermal filler injections in photodamaged human skin. Arch Dermatol 143(2):155-163 